\section{Epiphora as a sign of unexpected underlying squamous cell carcinoma within sinonasal inverted papilloma}

\author{
Alessandra Di Maria ${ }^{1}$, Filippo Confalonieri'1,2*, Raffaele \\ Piscopo ${ }^{1,2}$, Laura Balia ${ }^{1}$ and Luca Malvezzi ${ }^{1}$
}

${ }^{1}$ Humanitas Clinical and Research Center - IRCCS - Department of Ophthalmology, via Manzoni 56, 20089 Rozzano - Milan, Italy

${ }^{2}$ Humanitas University, Department of Biomedical Sciences, Via Rita Levi Montalcini 4, 20090

Pieve Emanuele - Milan, Italy

\section{Introduction}

Sinonasal inverted papilloma (SIP) is a benign tumor which originates from the sinonasal Schneiderian mucosa and accounts for $0.5 \%$ to $4 \%$ of all nasal and sinus neoplasm [1]. Pathologically, SIP epithelium inverts into submucosal stroma, which is different from other types of nasal papilloma. Unlike other benign tumors, SIP exhibits remarkable aggressive behaviors, including invasiveness, recurrence and malignant transformation [2]. Therefore, SIP can spread into the paranasal sinus, orbit, and cranial base, which can lead to poor prognosis for SIP patients [2]. Secondary squamous cell carcinoma is malignancy formation within the SIP. It is considered primary if there is no additional mucosal condition or secondary if it occurs in conjunction with an additional condition, which is estimated to occur in about $9 \%$ of cases [3]. The authors report the case of a 66-year-old woman with SIP and secondary SCC of the right nasal cavity. This study was adherent with the tenets of the Declaration of Helsinki.

\section{Case presentation}

A 66-year-old woman presented with a 4-year history of right-sided epiphora and new onset swelling at the right medial canthus mimicking a lacrimal sac's mucocele. She denied bloody tears, pain and diplopia. The patient was referred to the hospital eye service with a diagnosis of a chronic dacryocystitis and nasal obstruction.

The ophthalmologic examination revealed a firm, nontender, $1 \mathrm{~cm}$ mass palpable just below the right medial canthal ligament. Irrigation showed regurgitation of mucopurulent material. Uncorrected visual acuity was $7 / 9$ in both eyes. No RAPD nor proptosis were detected. No other palpable masses nor lymphadenopathy were present in the head and neck region.

\section{More Information}

*Address for Correspondence: Filippo Confalonieri, MD, Humanitas Clinical and Research Center - IRCCS - Department of Ophthalmology, via Manzoni 56, 20089 Rozzano - Milan, Department of Biomedical Sciences, Humanitas University, Italy, Tel: +393384563948; Email: filippo.confalonieri01@gmail.com

Submitted: 29 May, 2020
Approved: 12 June, 2020
Published: 15 June, 2020

How to cite this article: Di Maria A, Confalonieri F, Piscopo R, Balia L, Malvezzi L. Epiphora as a sign of unexpected underlying squamous cell carcinoma within sinonasal inverted papilloma. Arch Case Rep. 2020; 4: 038-040.

DOI: 10.29328/journal.acr.1001038

ORCiD: orcid.org/0000-0003-4079-8881 Copyright: (c) 2020 Di Maria A, et al. This is an open access article distributed under the Creative Commons Attribution License, which permits unrestricted use, distribution, and reproduction in any medium, provided the original work is properly cited.

\section{D) Check for updates \\ OPen Access}

Endoscopic examination of the nasal cavities showed a polypoid lesion of the right lateral wall and diffuse nonspecific mucosal edema.

CT scan of the orbit revealed $2 \times 2 \mathrm{~cm}$ well defined, heterogeneously enhancing abnormal soft tissue mass in the right nasolacrimal sac, duct and lateral wall of right nasal cavity. Bony erosion of the orbital rim was observed (Figure $1 \mathrm{~A}, \mathrm{~B})$.

MRI scan substantiated a T2 mildly hypointense, T1 isointense mass with erosion of the inferior orbital wall.

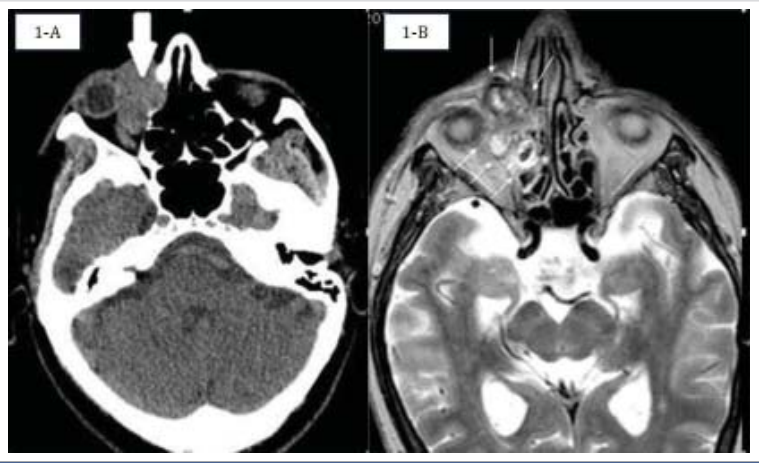

Figure 1: A: Non enhanced axial CT shows bone erosion of the papyracea lamina of ethmoid bone. B: Axial T2 weighted sequence shows an extraconal and intraconal mass (white arrows) in the right orbit, with non-homogeneous hyperintensity ed irregular margins. The lesion involves the nasolacrimal canal, the papyracea lamina and the anterior ethmoid cells. 
Endoscopic biopsy was carried out and the histological examination detected an inverted papilloma with synchronous squamous cell carcinoma (Figure 2).

A multidisciplinary approach is our preferred method of choice for cases with both nasal and orbital involvement. The surgical resection planning was a joint effort of the Ophthalmology and the Otorhinolaryngology departments.

The patient underwent lateral rhinotomy with orbitotomy. The orbital cavity was filled with a penduculated temporal muscle flap, covered with a dermo-epidermal skin graft (Thiersch graft).

Histological examination reported radical tumor excision and immunohistochemical staining was negative for Ki67 and HPV.

The patient was submitted to adjuvant radiotherapy after surgery. The total dosage of radiation was $60 \mathrm{~Gy}$ in 30 sessions. Endoscopic examinations and medications of the nasal fossa were performed every three months in the follow-up period. One year after surgery, both PET and MRI of the head and neck were negative for recurrent disease. During the following 4 years of follow-up, MRI was performed together with clinical examinations and neck ultrasound. No recurrence was detected.

\section{Discussion}

Sinonasal Inverted papilloma (SIP) is a rare benign sinonasal tumor, three characteristics make this tumor very different from other sinonasal tumors: a relatively strong potential for local destruction, high rate of recurrence, and a risk of carcinomatous evolution [4]. Unlike most papillomas of the body, this mucosal lesion inverts, rather than everts, into the underlying connective tissue stroma [1].

Clinical presentation is most commonly a male subject who is 50-69 years old. Classically, the "tumor" originates from the lateral nasal wall, particularly in the osteomeatal

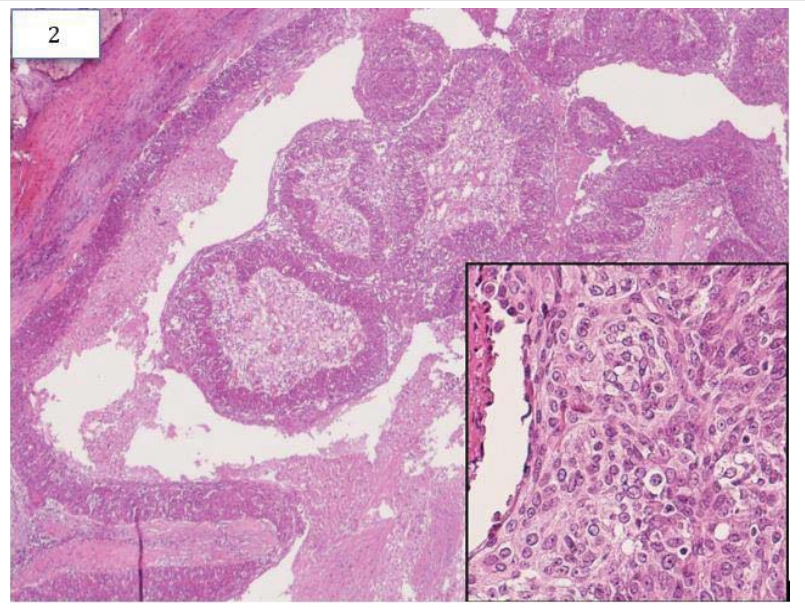

Figure 2: HE 10X and HE 40X complex area of the middle meatus, and adjacent inferior and middle turbinates, and subsequently involves the contiguous paranasal sinuses, with the maxillary sinus as the most frequent sinus involved and ethmoid sinus as the second most common. Unilateral nasal obstruction and intermittent epistaxis are the most common presenting symptoms [1].

Up to $90 \%$ of tumors of head and neck are squamous cell carcinomas (SCCs) [5], which represent the seventh most prevalent cancer worldwide. The male-to-female incidence ratio is $3: 1$, and about $70 \%$ of new cases occur in low- and middle-income countries. In 2018, there were an estimated 453000 deaths from head and neck cancer globally. In the past 15 years, strong evidence has accumulated that infection with certain human papillomaviruses (HPVs) is etiologically involved in a subset of head and neck cancers [6].

Squamous cell carcinoma may be a secondary malignancy originating within the SIP. It is considered primary if there is no additional mucosal condition or secondary if it occurs in conjunction with an additional condition, which is estimated to occur in about $9 \%$ of cases [3] SIP is usually not a primary lacrimal drainage system tumor but a primary tumor of the tissue surrounding the lacrimal drainage system and spreads to the lacrimal sac via secondary extension. It is exceedingly rare for SIP to originate from the lacrimal sac, having been reported only 7 times in the past 30 years [7].

To date, the treatment for SIP includes surgery and surgery combined with radiotherapy for SIP-associated squamous cell carcinoma (SIP/SCC). Currently, the majority of surgeons prefer endoscopic methods to traditional external approaches, due to similar success rates, less trauma and no facial scars.

However, the common view is that SIP recurrence is due to inadequate removal during the first surgery [8]. Therefore, preoperative evaluation as well as postoperative follow up is very important.

The present case points out the importance of including mass in differential diagnosis of nasolacrimal duct obstruction when epiphora is the only manifest sign.

Masses are uncommon but not a rare cause of nasolacrimal duct obstruction. Surgical teams performing high numbers of dacryocystorhinostomy procedures should be aware of such pathology and follow a systematic multidisciplinary approach. This is essential since neoplastic secondary acquired nasolacrimal duct obstructions (neoplastic SANDOs) can be easily mistaken for benign processes such as primary acquired nasolacrimal duct obstructions (PANDOs) [9].

We subject all candidates for DCR surgery to a preoperative dacyo-CT scan to exclude facial bone anomalies and concomitant tear duct stenosis.

We believe that dacryo-CT scan is the most useful exam for evaluation of the lacrimal drainage system, together with nasal cavities and sinuses. 
This case demonstrates that bone destruction with intraorbital and intracranial extension are possible severe complications.

Whenever a malignant tumor expands beyond the lacrimal sac, it should be treated according to the degree of histological pattern and extension within the orbital soft tissue. The major cause for recurrence of the disease, or rather failure to cure it, is in case of incomplete resection of the tumor or insufficient cleanliness of the surgical margin [9].

Every attempt should be made to remove the whole tumor during primary surgery. To obtain neoplastic radicality, we proceeded with orbital exenteration combined with lateral rhinotomy. It is intuitive that radical surgery can be most easily performed in the initial stages of the disease. Our patient reported having just persistent epiphora for at least 1 years prior to presentation of nasal symptoms, when she also noted that a right medial canthal swelling had recently arisen. Therefore, unilateral persistent epiphora in patient at risk must be taken seriously.

Recurrence rate for inverted papilloma has been described in up to $78 \%$ in the literature and some risk factors for recurrence were identified, such as tobacco exposure, size of tumor, high hyperkeratosis, squamous hyperplasia, increased number of mitosis, HPV positivity and tumor location. In most cases, recurrence occurs in the first three years after surgery, although, there are reports of recurrence after 10 years [10]. So, every attempt should be made to remove the whole tumor during primary surgery and long-term follow-up is required in order to rule out recurrences. In our case both Ki-67 and HPV were negative and our five year follow-up did not reveal any recurrence.

Because of the rarity of the tumors, we believe it is advisable that such cases be managed in a multidisciplinary setting through a systematic approach.

\section{References}

1. Wood JW, Casiano RR. Inverted papillomas and benign nonneoplastic lesions of the nasal cavity. Am J Rhinol Allergy. 2012; 26: 157-163. PubMed: https://www.ncbi.nlm.nih.gov/pubmed/22487294

2. Kim DY, Hong SL, Lee CH, Jin HR, Kang JM, et al: Inverted papilloma of the nasal cavity and paranasal sinuses: A Korean multicenter study. Laryngoscope. 2012; 122: 487-494.

PubMed: https://www.ncbi.nlm.nih.gov/pubmed/22253070

3. Nygren A, Kiss K, von Buchwald C, Bilde S. Rate of recurrence and malignant transformation in 88 cases with inverted papilloma between 1998-2008, Acta Oto-Laryngologica. 2016; 136: 3: 333-336. PubMed: https://www.ncbi.nlm.nih.gov/pubmed/26838581

4. Lisan $Q$, Laccourreye $O$, Bonfils $P$. Sinonasal Inverted Papilloma: From Diagnosis to Treatment Eur Ann Otorhinolaryngol Head Neck Dis. 2016;133: 337-341.

PubMed: https://www.ncbi.nlm.nih.gov/pubmed/27053431

5. Koh J, Walsh P, D'Costa I, Bhatti O. Head and neck squamous cell carcinoma survivorship care. Aust J Gen Pract. 2019; 48: 846-848. PubMed: https://www.ncbi.nlm.nih.gov/pubmed/31774982

6. Wild CP, Weiderpass E, Stewart BW, Editor. World Cancer Report: Cancer Research for Cancer Prevention. Lyon, France: International Agency for Research on Cancer. http://publications.iarc.fr/586

7. Hardy AW, Dwivedi RC, Masterson L, Riffat F, Marker A, et al. Inverted papilloma of lacrimal sac invading into the orbit: Case report and review of literature. J Cancer Res Therapeut. 2015; 11: 238-240.

8. Hildenbrand T, Weber R, Merten s J, Stuck BA, Hoch S, et al. Surgery of Inverted Papilloma of the Maxillary Sinus via Translacrimal ApproachLong-Term Outcome and Literature Review. J Clin Med. 2019; 8: 1873. PubMed: https://www.ncbi.nlm.nih.gov/pmc/articles/PMC6912689/

9. Xiao-Ting W, Peng L, Xiu-Qing W, Hai-Bo W, Wen-Hui P, et al Factors affecting recurrence of sinonasal inverted papilloma. Eur Arch Otorhinolaryngol. 2013; 270: 1349-1353.

PubMed: https://www.ncbi.nlm.nih.gov/pubmed/23064461

10. Sbrana MF, Borges RFR, Pinna FR, Neto DB, Voegels RL, et al Sinonasal inverted papilloma: rate of recurrence and malignant transformation in 44 operated patients. Braz J Otorhinolaryngol. 2019; S1808-8694(19)30088-6.

PubMed: https://www.ncbi.nlm.nih.gov/pubmed/31455578 